\title{
Concentrations of metalloproteinase-1 in patients with morphea treated with phototherapy: a preliminary study
}

\author{
Anna Malewska-Woźniak, Martha Lodyga, Zygmunt Adamski \\ Department of Dermatology, Poznan University of Medical Sciences, Poznan, Poland
}

Adv Dermatol Allergol 2022; XXXIX (5): 972-975

DOI: https://doi.org/10.5114/ada.2021.113127

\begin{abstract}
Introduction: Morphea (localized scleroderma) is a rare, chronic, inflammatory connective tissue disease, characterized by immune system dysfunction, vasculopathy and skin fibrosis. One of the most effective treatments is phototherapy. Phototherapy has been found to be effective in treating localized scleroderma by inducing the expression of metalloproteinase-1.

Aim: To compare the concentrations of metalloproteinase (MMP-1) before psoralen and ultraviolet A (PUVA) and ultraviolet A1 (UVA1) treatments in the serum of patients with morphea.

Material and methods: The observational study was conducted in one research centre and included patients with generalised morphea who were treated with PUVA and UVA1 phototherapies. The mean age of all morphea patients included in the study was 55.7 years. The levels of MMP-1 were examined by ELISA (The Biorbyt Human MMP-1 ELISA - Enzyme-Linked Immunosorbent Assay).

Results: The study showed that patients treated with PUVA and UVA1 had an improvement based on clinical measures, resulting in a reduction of clinical score. However, we did not observe statistically significant differences in MMP-1 concentrations before and after treatment. Limitations: The study sample was relatively small. Further studies on a larger group of patients would be beneficial.

Conclusions: Our data suggest that there is a possible correlation between MMP-1 concentrations and phototherapy. MMP-1 levels were found to be increased following phototherapy treatment, which may suggest a correlation with better response to treatment in patients with morphea. However, further research is needed.
\end{abstract}

Key words: morphea, localized scleroderma, phototherapy, ultraviolet A1, UVA1, psoralen and ultraviolet A, PUVA, matrix metalloproteinase-1.

\section{Introduction}

Morphea, otherwise known as localized scleroderma, is a chronic, autoimmune disease with an unknown aetiology. It is characterized by the involvement of the skin, subcutaneous tissue and deeper tissues. There are three clinical phases: inflammatory, progressive tissue sclerosis, and atrophic (Figures 1-3). Assessment of disease progression and tissue damage is based on a validated scale proposed by the Localized Scleroderma Clinical and Ultrasound Study Group. The LS Cutaneous Assessment Tool (LoSCAT) is an accessible, repeatable, and sensitive scale, which can be used to monitor the effects of treatment. It is a combination of the three indices: LS SkinSeverity Index (LoSSI) for the assessment of disease activity and severity, LS Skin Damage Index (LoSDI) for the assessment of tissue damage and the physician's global assessment (PGA), which is a general patient assessment [1-3].

Most patients with mild skin involvement during the course of morphea require topical treatment with glucocorticoids, calcineurin inhibitors, calcipotriol or phototherapy (UVA/PUVA). More severe forms of morphea that are characterized by extensive tissue involvement, require systemic therapy with oral glucocorticosteroids, methotrexate, or mycophenolate mofetil and treatment should be started as soon as possible [4].

Ultraviolet A1 (UVA1) and psoralen and ultraviolet A (PUVA) are phototherapy interventions recommended by the Polish Dermatological Society for the treatment of patients with morphea $[4,5]$. Phototherapy works directly on the three stages of morphea lesions by restoring the balance of the immune system, reducing collagen pro-

Address for correspondence: Anna Malewska-Woźniak, Department of Dermatology, Poznan University of Medical Sciences, Poznan, Poland, phone: +48 608523 020, e-mail: annamalewskawozniak@gmail.com Received: 8.12.2021, accepted: 29.12.2021. 


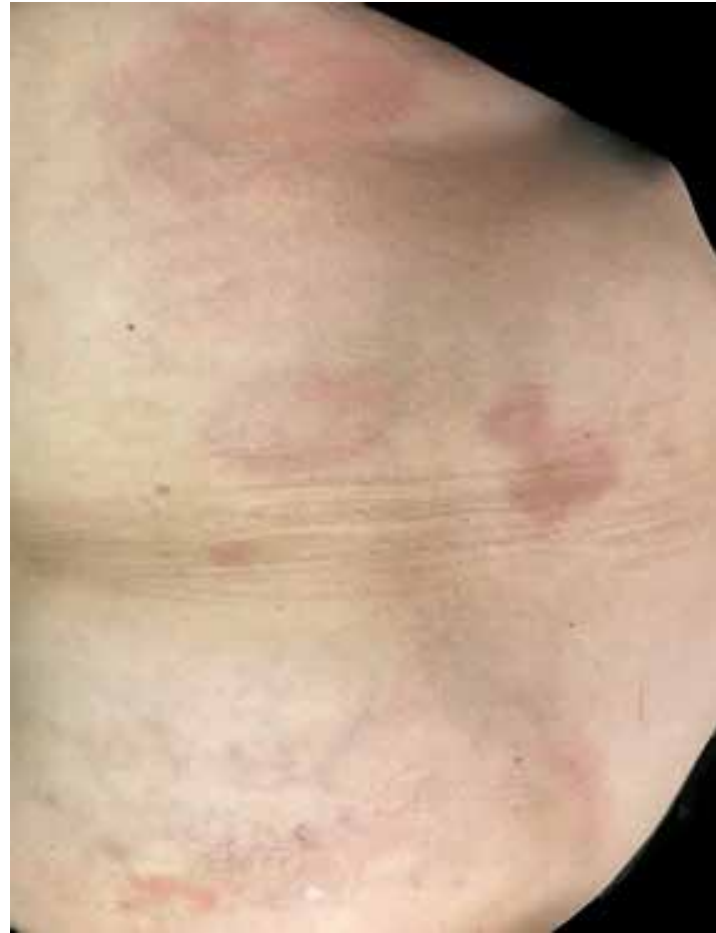

Figure 1. Clinical stages of morphea - inflammation

duction, and neovascularization [5]. PUVA photochemotherapy combines psoralen with long-wave UV radiation (UVA) [6]. UVA penetrates the dermis, where it increases the production of metalloproteinases, which leads to collagen degradation. It is through this mechanism that phototherapy is used to treat morphea $[7,8]$. PUVA shows the greatest efficacy in cases of early inflammatory lesions secondary to localized scleroderma [4, 5]. UVA1 phototherapy (320-400 nm) is a new, but commonly used treatment for localized scleroderma [5]. UVA1 affects various stages of the disease process in morphea by inhibiting the inflammatory process, progression of the disease, and ultimately, the process of fibrosis. It also influences the production of collagenases by fibroblasts. The increased enzyme expression correlated with clinical improvement in morphea patients [9, 10].

Matrix metalloproteinase-1 (MMP-1) is also known as an interstitial and fibroblast collagenase. Collagenases are zinc-dependent proteases that play an essential role in tissue remodelling. MMP-1 plays an important role in the degradation of collagen in inflammatory diseases like morphea [11]. Phototherapy such as UVA1 induces production of MMP-1 and thusly, collagen degradation [12].

\section{Aim}

The aim of this study was to compare the serum concentrations of MMP-1 in patients with the diagnosis of morphea before and 3 months after phototherapy.

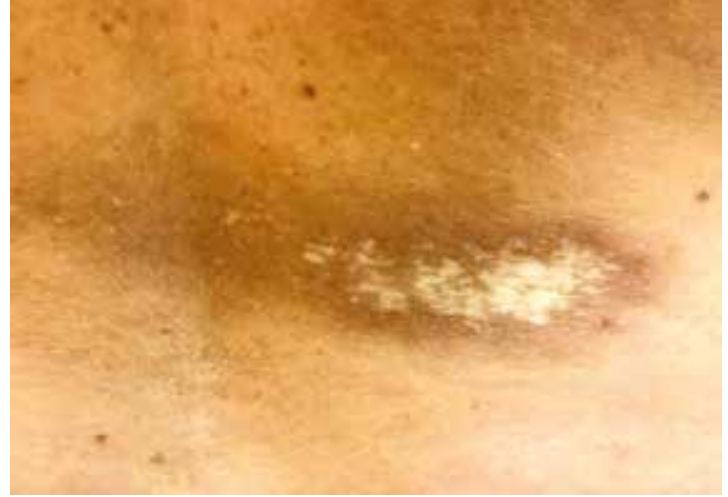

Figure 2. Clinical stages of morphea - sclerosis

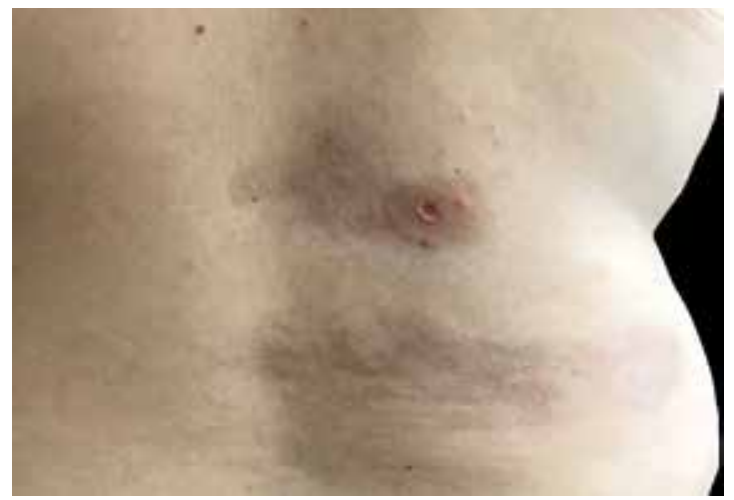

Figure 3. Clinical stages of morphea - atrophy with hyperpigmentation and scar after biopsy taken

\section{Material and methods}

Patients with morphea who were treated with phototherapy (PUVA, UVA1) were included in the study group. The diagnosis of morphea was confirmed by histologic examination. All patient phototherapy notes and case records were reviewed. The data collected included age, sex, treatment parameters, results of the LS Cutaneous Assessment Tool (LoSCAT), concentrations of MMP-1 in patients' serum before and after 3 months of phototherapy treatments. The detection and quantification of MMP-1 was examined by ELISA (The Biorbyt Human MMP-1 ELISA - Enzyme-Linked Immunosorbent Assay).

The study was performed in accordance with the ethical standards of the local institution committee (Bioethics Commission approval number 525/18).

\section{Statistical analysis}

Variables are described by the parameters of descriptive statistics (i.e. arithmetic mean, standard deviation, median, minimum and maximum value, lower and upper quartile).

The normality of the distribution was tested with the Shapiro-Wilk test. If the data met the assumption, then the dependencies were analysed using the Pearson corre- 
lation, otherwise, the Spearman correlation was used. The difference of MMP-1 was analysed by the Mann-Whitney test. Student's t-test for dependent groups was used to compare the levels of MMP-1 and LOSCAT before and after.

For the performed statistical analyses, the significance level was 0.05. The analyses were performed using the Statistica 12 program.

\section{Results}

The mean age of all morphea patients included in the study was 55.7 years. The youngest patient was 34 years and the oldest was 74 years old. The study group was only comprised of women. Ultimately, 10 patients were included in this trial. From the total, 5 patients were treated with PUVA sessions three times per week with up to 20 radiations. Whereas, the remaining 5 patients received low to medium doses of UVA1 $\left(20-50 \mathrm{~J} / \mathrm{cm}^{2}\right)$ phototherapy sessions with a range of $20-40$ radiations in total. These treatment sessions were delivered five times per week.

A significant reduction in LoSCAT scores was observed in both groups of patients after 3 months of phototherapy treatments (Figure 4). The mean concentration of MMP-1 before treatment was $1662.03 \mathrm{pg} / \mathrm{ml}$ and $2257.04 \mathrm{pg} / \mathrm{ml}$ after phototherapy.

However, there was no statistically significant difference between the concentrations of MMP-1 before and after 3 months of phototherapy treatments $(p=0.1441)$ (Figure 5). Furthermore, we did not confirm any statistical differences between the study groups. We were not

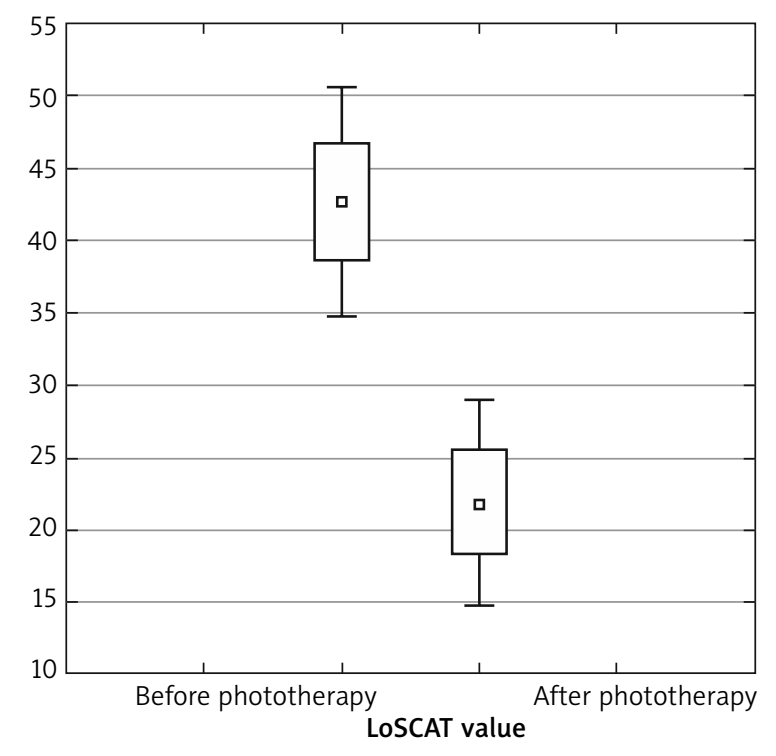

-Mean

$\square$ Mean \pm standard error

I Mean $\pm 1.96 \times$ standard error

Figure 4. LOSCAT values before and after phototherapy in patients with morphea $(p=0.0001)$ able to prove superiority of one phototherapy method over the other based upon MMP-1 concentrations ( $p=$ 0.9519). Additionally, the study did not show that the age of patients affected MMP-1 concentrations after phototherapy treatments $(p=0.1430)$.

\section{Discussion}

Phototherapy treatment for morphea was first reported in 1994 with the successful use of PUVA therapy by Kerscher. Shortly after, benefits of UVA1 therapy were demonstrated in patients with morphea. As to date, there have been no studies comparing superiority between UVA1 and PUVA therapies and correlation between the concentrations of MMP-1. In our study, 5 of the total 10 patients received low to medium doses of UVA1 (20-50 J/ $\left.\mathrm{cm}^{2}\right)$ phototherapy with treatment sessions ranging between 20 and 40 sessions. Treatment was delivered five times per week. The remaining 5 patients were treated with three times per week PUVA session with up to 20 radiations. Clinical improvement was observed to be significant in both UVA1 and PUVA-treated patients when utilizing LoSCAT evaluation. However, we were unable to prove the advantage of one method over the other.

Increased concentrations of MMP-1 after phototherapy treatments confirm its effectiveness in morphea, however, the values were not statistically significant. In a study conducted by Tomimura et al., elevated levels of antibodies against matrix metalloproteinase- 1 were found in patients with localized scleroderma. This study

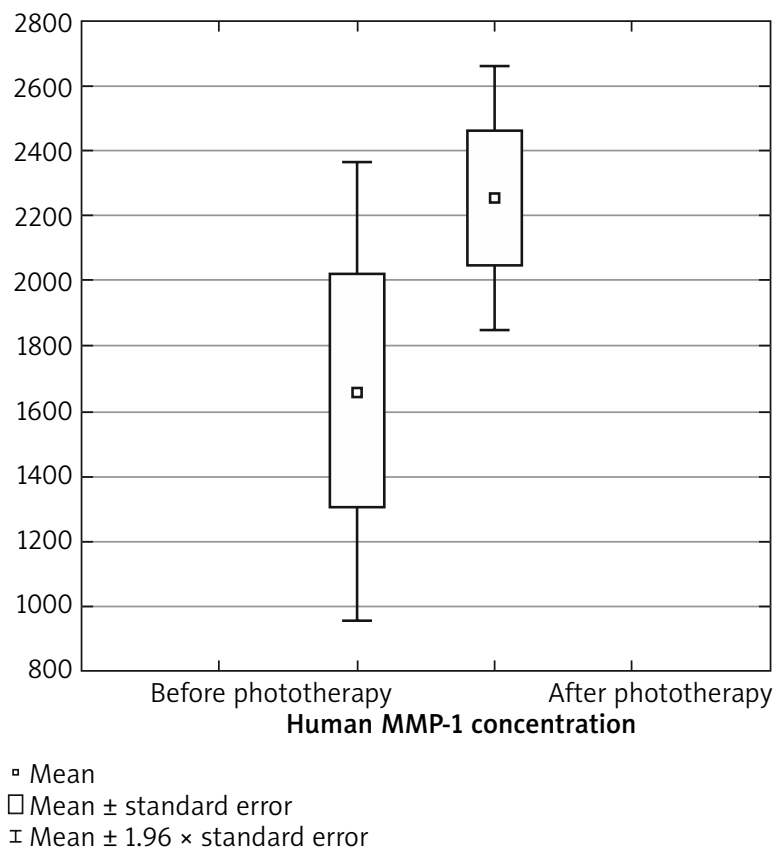

Figure 5. Concentrations of MMP-1 before and after phototherapy $(p=0.1441)$ 
suggested that anti-MMP-1 autoantibody may be a new antibody responsible for morphea. The high serum antibody concentrations suggest increased enzyme activity and thus, increased collagen degradation [13]. Another study found an increased expression of MMP-1 and MMP-3 after high-dose UVA1 treatments and a reduction in collagen type I and collagen type II expressions was also observed. That study demonstrated that UVA1 not only prevented the progression of dermal fibrosis, but also induced a regression of fibrotic changes due to degradation of collagen [14]. MMP-1 induction is one of the effects of UVA light which enables degradation of various collagens $[12,15,16]$. These phototherapy mechanisms are used to treat morphea. Increased MMP-1 expression is one of the most important indicators for successful UVA1 treatment and significantly improves the clinical condition of LS [17]. MMP-1 is specifically responsible for the breakdown of collagen [18]. Therefore, a correlation can be expected in a better clinical response and a higher concentration of MMP-1. In our study, increased MMP-1 values are not statistically significant. We found no statistically significant correlation between treatment response and MMP-1 levels. We are confident that further research in larger groups would be beneficial. Our research is an introduction to further research.

\section{Conclusions}

Morphea is a cosmetic problem for patients as it significantly reduces their quality of life. It should be distinguished from systemic scleroderma in which internal organs are affected. Early detection and implementation of appropriate treatment is of great importance in preventing permanent sclerosis of the skin. Morphea is an inflammatory, autoimmune, sclerotic skin disease that can also affect the underlying soft tissues. Although the cause of morphea remains poorly understood, attention is focused on genetic predisposition, immune dysregulation, and environmental factors [1-4]. The main indication for UVA1 phototherapy treatment is sclerosing skin disorders especially morphea. Similarly, PUVA therapy has excellent effects in morphea patients [5]. Our study suggests that increased MMP-1 values before and after the intervention with phototherapy are not statistically significant, but higher concentrations of MMP-1 cause increased degradation of collagen after phototherapy. Thusly, preventing skin from sclerosis and further consequences of the disease with progression. UVA1 and PUVA reduce dermal thickness and sclerosing by inducing the expression of MMP-1 in the blood serum of patients with morphea. We were also able to prove the importance and crucial role phototherapy plays in the treatment of patients with morphea.

Although we had a relatively small sample size in our study, we treat our research as a preliminary study. Further studies comprised of a larger group of patients would be beneficial and promising.

\section{Conflict of interest}

The authors declare no conflict of interest.

\section{References}

1. Fett N, Werth VP. Update on morphea. Part I. Epidemiology, clinical presentation, and pathogenesis. J Am Acad Dermatol 2011; 64: 217-28.

2. Fett N, Werth VP. Update on morphea. Part II. Outcome measures and treatment. J Am Acad Dermatol 2011; 64: 231-42.

3. Wolska-Gawron K, Krasowska D, Localized scleroderma classification and tools used for the evaluation of tissue damage and disease activity/severity. Dermatol Rev 2017; 104: 269-89.

4. Wolska-Gawron K, Michalska-Jakubus M, Krasowska D. Localized scleroderma - current treatment options. Dermatol Rev 2017; 104: 606-18.

5. Placek W, Kaszuba A, Lesiak A, et al. Phototherapy and photochemotherapy in dermatology. Recommendations of the Polish Dermatological Society. Dermatol Rev 2019; 106: 237-56.

6. Schneider LA, Hinrischs R, Scharffetter-Kochanek K. Phototherapy and photochemotherapy. Clin Dermatol 2008; 26: 464-76.

7. Chaowattanapanit S, Choonhakarn C, Foocharoen C, Julanon N. Phototherapy in systemic sclerosis: review. Photodermatol Photoimmunol Photomed 2017; 33: 296-305.

8. Suganuma K, Nakajima H, Ohtsuki M, Imokowa G. Astaxanthin attenuates the UVA-induced up-regulation of matrixmetalloproteinase-1 and skin fibroblast elastase in human dermal fibroblast. J Dermatol Sci 2010; 58: 136-42.

9. Gruss C, Reed JA, Altmeyer P, et al. Induction of interstitial collagenase (MMP-1) by UVA-1 phototherapy in morphea fibroblasts. Lancet 1997; 350: 1295-6.

10. York NR, Jacobe HT. UVA1 phototherapy: a review of mechanism and therapeutic application. Int J Dermatol 2010; 49: 623-30.

11. Nagase H, Woessner JF. Matrix metalloproteinases. J Biol Chem 1999; 274: 21491-4.

12. Nisar MF, Parsons KSG, Bian CX, Li Zhong J. UVA irradiation inducesd heme oxygenase-1: a novel phototherapy for morphea. Photochem Photobiol 2015; 91: 210-20.

13. Tomimura S, Ogawa FM, Iwata Y, et al. Autoantibodies againts matrix metalloproteinase- 1 in patients with localised scleroderma. J Dermatol Sci 2008; 52: 47-54.

14. Karpec D, Rudys R, Leonaviciene L, et al. The impact of highdose narrowband ultraviolet A1 on dermal thickness, collagen and matrix-metalloproteinases in animal model of scleroderma. J Photochem Photobiol B 2017; 173: 448-55.

15. Wlaschek M, Heined G, Poswig A, et al. UVA-induced autocrine stimulation of fibroblast-derived collagenase/MMP1 by interrelated loops of interleukin-1 and interleukin-6. Photochem Photobiol 1994; 59: 550-6.

16. Tewari A, Dafou D, Grys K, et al. Matrix metalloproteinase (MMP) 12 is a UVA1 specific biomarker. J Invest Dermatol 2013; 133: S209-221.

17. Stege H, Berneburg M, Humke S, et al. High-dose UVA1 radiation therapy for localized scleroderma. J Am Acad Dermatol 1997; 36: 938-44.

18. Arndt S, Lissner C, Unger P, et al. Biological effects of a new ultraviolet A1 prototype based on light-emitting diodes on the treatment of localised scleroderma. Exp Dermatol 2020; 29: 1199-208. 